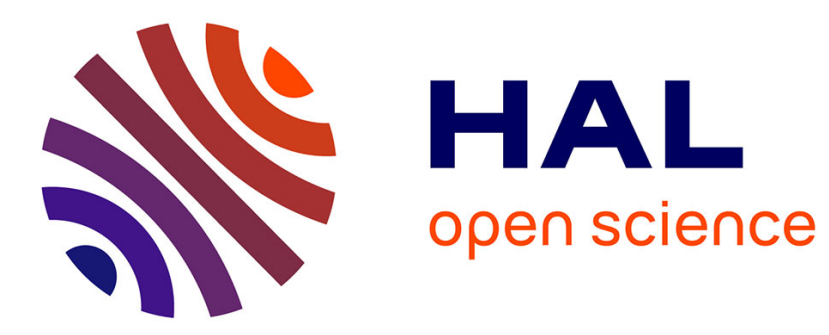

\title{
Châteaux et enceintes des Vosges du Nord. Topographie et longue durée
}

\author{
Jean-Jacques Schwien
}

\section{To cite this version:}

Jean-Jacques Schwien. Châteaux et enceintes des Vosges du Nord. Topographie et longue durée. Archimède: archéologie et histoire ancienne, 2016, 3, pp.37-48. 10.47245/archimede.0003.ds1.05 . hal-01587231

\section{HAL Id: hal-01587231 \\ https://hal.science/hal-01587231}

Submitted on 20 Sep 2017

HAL is a multi-disciplinary open access archive for the deposit and dissemination of scientific research documents, whether they are published or not. The documents may come from teaching and research institutions in France or abroad, or from public or private research centers.
L'archive ouverte pluridisciplinaire HAL, est destinée au dépôt et à la diffusion de documents scientifiques de niveau recherche, publiés ou non, émanant des établissements d'enseignement et de recherche français ou étrangers, des laboratoires publics ou privés. 


\section{ARCHIMĖDE}

ARCHÉOLOGIE ET HISTOIRE ANCIENNE

\section{DOSSIER THÉMATIQUE : DES FOSSÉS ET DES REMPARTS. ENCEINTES ET SITES FORTIFIÉS DU RHIN SUPÉRIEUR ENTRE PROTOHISTOIRE ET MOYEN ÂGE}

\section{Olivier BUCHSENSCHUTZ}

Avant-propos. Des enceintes en terre anhistoriques à Google Earth

8 Lizzie SCHOLTUS

Histoire de la recherche dans le bassin de Saint-Dié-des-Vosges

20 Maxime WALTER

Les sites de hauteur du massif vosgien. Actualisation des données et modalités d'implantation

37 Jean-Jacques SCHWIEN Chateaux et enceintes des Vosges du Nord. Topographie et longue durée

49 Anne-Marie ADAM

La palissade dans tous ses états : I'enclos du Britzgyberg (Illfurth, Haut-Rhin) et autres aménagements palissadés dans les habitats du premier âge du Fer

60 Clément FÉLIU

L'enceinte inférieure du Frankenbourg (67) et les remparts à poteaux frontaux de la fin de l'âge du Fer dans l'espace du Rhin supérieur. Pour une révision de la typologie des Pfostenschlitzmauern

74 Jacky $\mathrm{KOCH}$ et Thomas FISCHBACH

Enceintes de hauteur en pierres et formes « primitives » de châteaux ? L'exemple du Bernstein

87 Adrien VUILLEMIN

Les enceintes urbaines en moyenne Alsace (1200-1850)

102 Jean-François PININGRE

Les enceintes de l'âge du Bronze et du premier âge du Fer en Franche-Comté. Un bilan des recherches

124 Clément FÉLIU et Jean-Jacques SCHWIEN

Conclusion. Nouvelles perspectives sur les enceintes du Rhin supérieur

\section{ACTUALITÉ DE LA RECHERCHE : ARCHÉOLOGIE DES RÉSEAUX}

\section{Claire CAMBERLEIN}

Les réseaux en archéologie : approche historiographique et interdisciplinaire

135 Thomas HUTIN

Lieux d'échanges et espaces publics en Gaule à La Tène finale

150 Streeve GENTNER

Économie du fer et voies de communication, de l'abattage du minerai à la distribution

du métal : I'exemple du nord de la Forêt-Noire au Ve siècle av. J.-C.

169 Loup BERNARD et Rémy WASSONG

Du Danemark au Fossé rhénan. Un siècle d'analyse des voies de communications protohistoriques : évolution des méthodes et mise en commun des données

184 Steeve GENTNER et Rémy WASSONG

Conclusion. L'archéologie des réseaux : une thématique aux multiples facettes

\section{VARIA}

187 Fábio VERGARA CERQUEIRA

To march in phalanx, to jump with weights, to tread the grapes, to knead the bread. What is the aulos for?

206 Hermann AMON

Les supra-commandements comme solution à la crise militaire du III siècle de l'Empire romain sous Philippe l'Arabe et Gallien

218 Martina BONO

Il processo di Cremuzio Cordo in Dio LVII, 24, 2-4

\section{LA CHRONIQUE D'ARCHIMÈDE}

228 Frédéric COLIN (éd.)

La Chronique d'Archimède. Bilan des activités scientifiques 2015-2016 de I'unité mixte de recherche 7044 


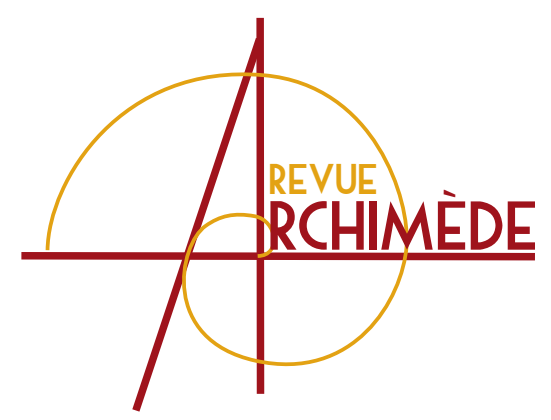

\section{CHÂTEAUX ET ENCEINTES DES VOSGES DU NORD. TOPOGRAPHIE ET LONGUE DURÉE}

Jean-Jacques SCHWIEN

Maître de conférences en archéologie médiévale

Université de Strasbourg UMR 7044 Archimède

schwien@unistra.fr

\section{RÉSUMÉ}

Parmi les questions portant sur les structures défensives figure toujours en bonne place celle du choix de l'implantation. Les pistes évoquées sont généralement liées à la topographie (éminences, éperons, îles...), aux réseaux de circulation (routes, vallées, cols...), aux densités et nature du peuplement (habitats, sanctuaires, marchés...) sans oublier la continuité des occupations avec le réaménagement de lieux déjà choisis anciennement. Cette démarche conduit souvent à répondre à partir du corpus existant, ne tenant compte que du choix définitif des constructeurs sans questionner le potentiel des pistes possibles à un moment donné. À partir de la fenêtre des Vosges du Nord, nous proposons ici de lister les critères généraux des choix possibles, avant tout liés à la topographie; mais nous intégrerons également la variable chronologique en comparant les situations en termes de modalités de peuplement sur la longue durée.
Among the questions carrying about the defensive structures always appears in good place that of the choice of the establishment. The evoked tracks are generally related to the geography (eminences, spurs, islands...), with the networks of circulation (roads, valleys, saddles...), with the densities and nature of the settlement (habitats, sanctuaries, markets...) without forgetting the inertia of the occupations with the refitting of already selected in the past places. This approach often results in answering starting from the existing corpus, taking account only final choice of the constractors without opening the question with the potential possible tracks at a given time. From the window of the north of the Vosges, we propose here to list the general criteria of the possible choices, above all related to topography; but we will also integrate the chronological variable by comparing the situations in terms of methods of settlement over the long time.

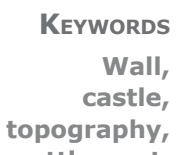


Les raisons de l'implantation d'un site défensif en un lieu donné font toujours partie des questionnements de ceux qui les étudient. Les conditions en sont multiples mais peuvent être regroupées en deux grandes familles : I'environnement naturel et le rapport aux communautés d'habitants. Comme la documentation primaire est le plus souvent muette sur ces questions, les chercheurs développent généralement un argumentaire de type régressif, considérant - de façon implicite ou non - que le lieu qui a été choisi était le meilleur ou le plus adapté à la situation. Il est plus difficile de se projeter en amont de ces réalisations pour savoir quel a été le potentiel des sites disponibles à un moment donné.

Dans le cadre de la réflexion de notre équipe sur le phénomène des sites de hauteur sur le temps long, nous proposons ici des pistes que nous espérons dynamiques. L'analyse croisée des données de la topographie et des connaissances sur l'état du peuplement devrait permettre sinon d'apporter toujours des réponses, du moins de mettre au point un premier catalogue de paramètres. Pour ce faire, il nous a semblé judicieux de travailler sur une fenêtre, en l'occurrence les Vosges du Nord, entre Wissembourg et Saverne. Cet espace est pour l'instant le plus propice à une analyse sur la longue durée, puisqu'une mise au

[1] WALTER 2016, dans ce même volume.

[2] La prospection, en effet, n'a pas été systématique sur le territoire en question, puisque pour l'instant, nous ne disposons pas ici d'une couverture Lidar qui seule permettrait de repérer des anomalies encore non signalées.

[3] On sait aujourd'hui qu'il y a de nombreuses enceintes de hauteur construites ou reprises entre I'Antiquité tardive et le premier Moyen Âge. Voir Steuer-Bierbrauer 2008. point vient d'y être faite sur les enceintes, dans la cadre du master de Maxime Walter [1], tout en étant également un secteur très riche en châteaux. Après avoir présenté notre corpus, nous traiterons ainsi successivement les questions de topographie (comparaison de l'altitude des sites) puis celles des paramètres du peuplement (analyse de la densité des occupations «périphériques »).

\section{LE CORPUS DES SITES}

Les enceintes retenues pour notre fenêtre d'étude à partir des recherches de Maxime Walter sont au nombre de 16. Rappelons que son travail, fondé sur la documentation publiée et des prospections systématiques sur ces lieux déjà signalés [2], a éliminé plusieurs sites trop rapidement retenus comme enceintes par des chercheurs des $\mathrm{XIX}^{\mathrm{e}}$ et début $X X^{e}$ siècles, mais en a confirmé d'autres. Parmi ces derniers, certains ont fait l'objet de recherches approfondies (relevés en plan et fouilles), comme le Fossé des Pandours ou la Heidenstadt, livrant ainsi des données solides en termes de chronologie. Pour la plupart des autres, tout en présentant des vestiges qualifiés de défensifs (murs en pierres sèches, fossés), les données chronologiques sont toutefois imprécises voire totalement absentes, ce qui nous laisse un large champ des possibles entre le Néolithique et le milieu du Moyen Âge [3]. Même si parfois il ne s'agit que du barrage d'un éperon, nous les appellerons donc ici « enceintes », sans aller jusqu'à reprendre I'ancien qualificatif « d'anhistorique », ici peu pertinent puisque certains sites sont datés (fig. 1). 


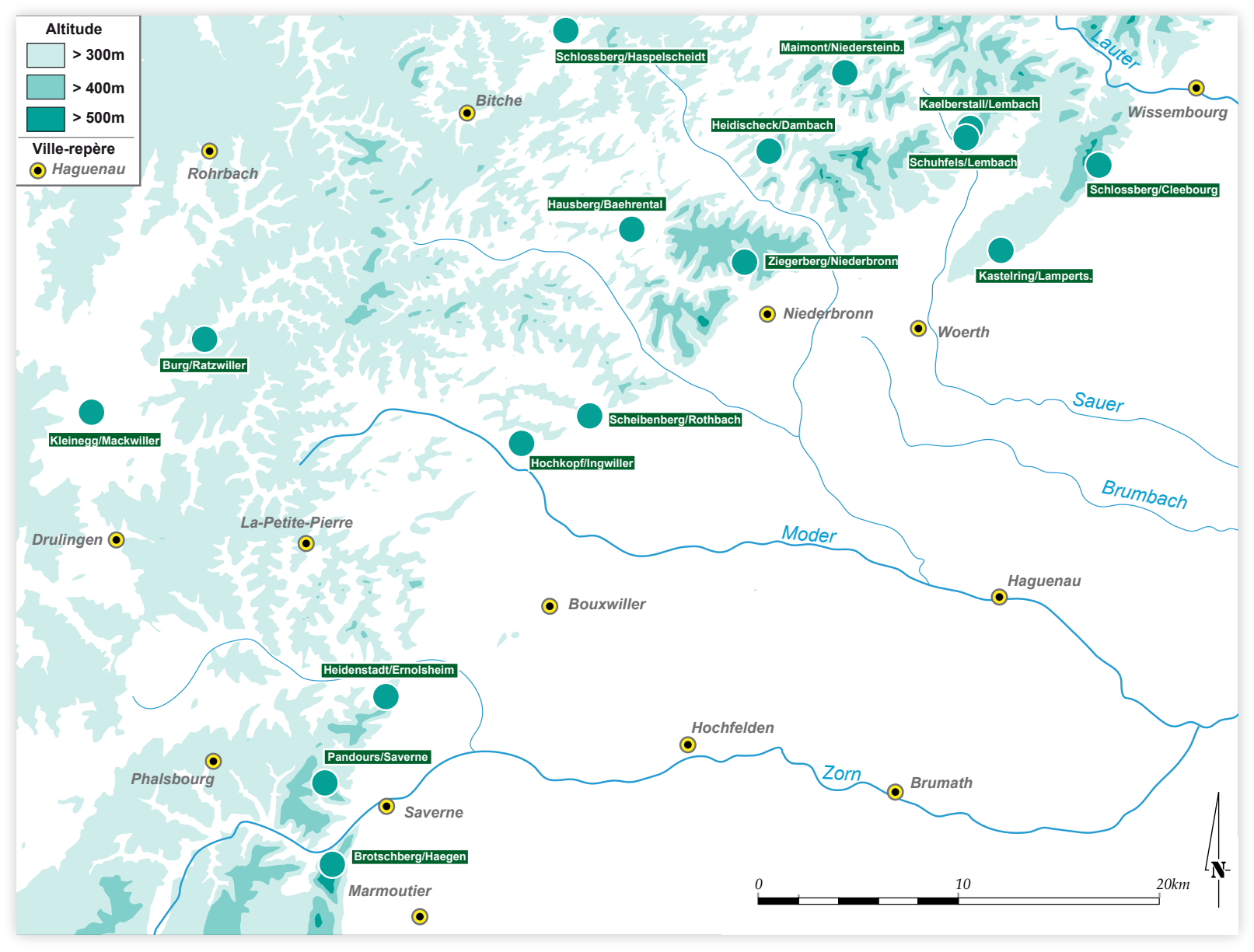

A Figure 1 : carte de localisation des enceintes.

(Sauf mention contraire, les figures sont de l'auteur)

$\checkmark$ Figure 2 : carte de localisation des sites de hauteur antiques.

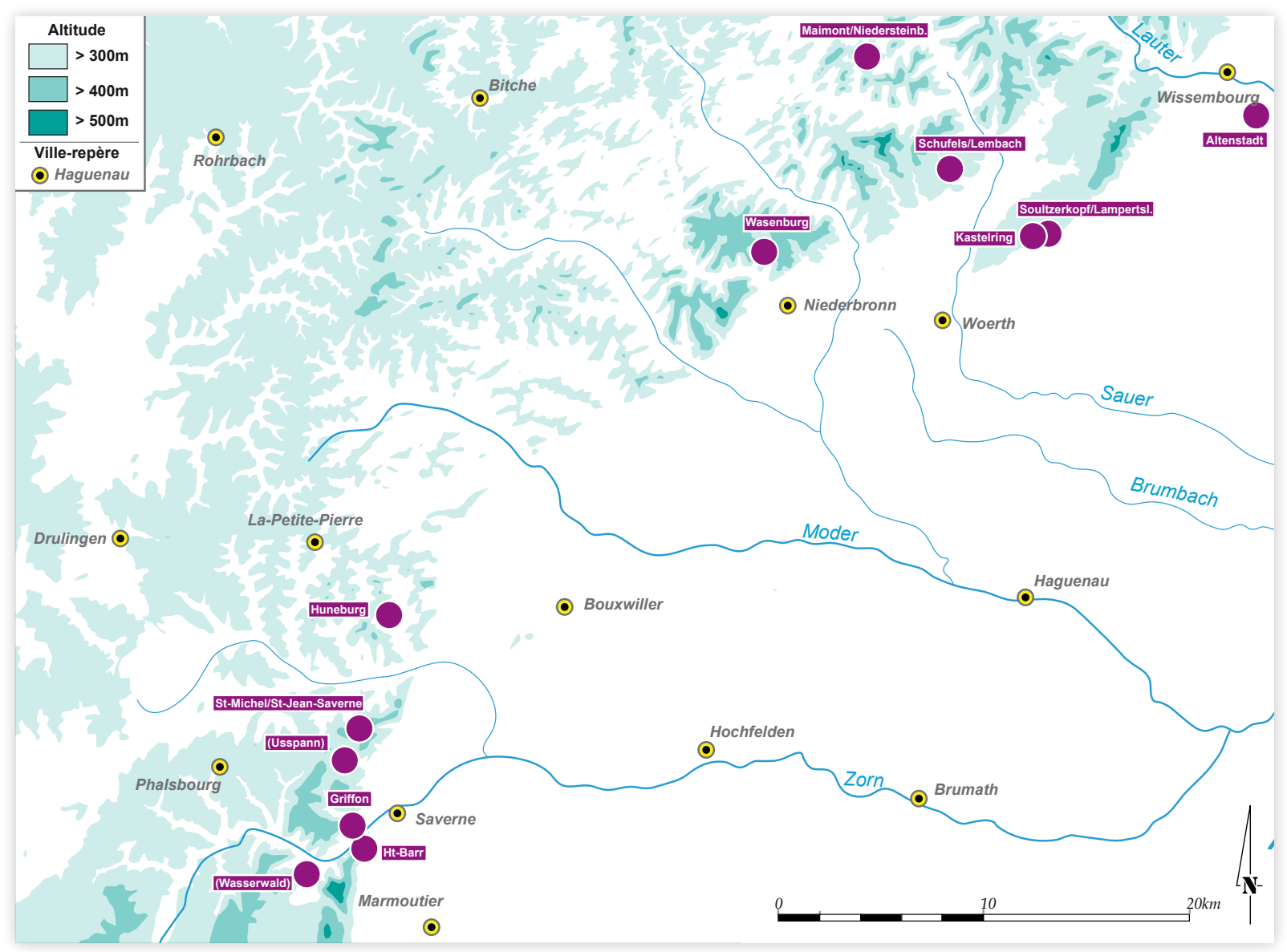




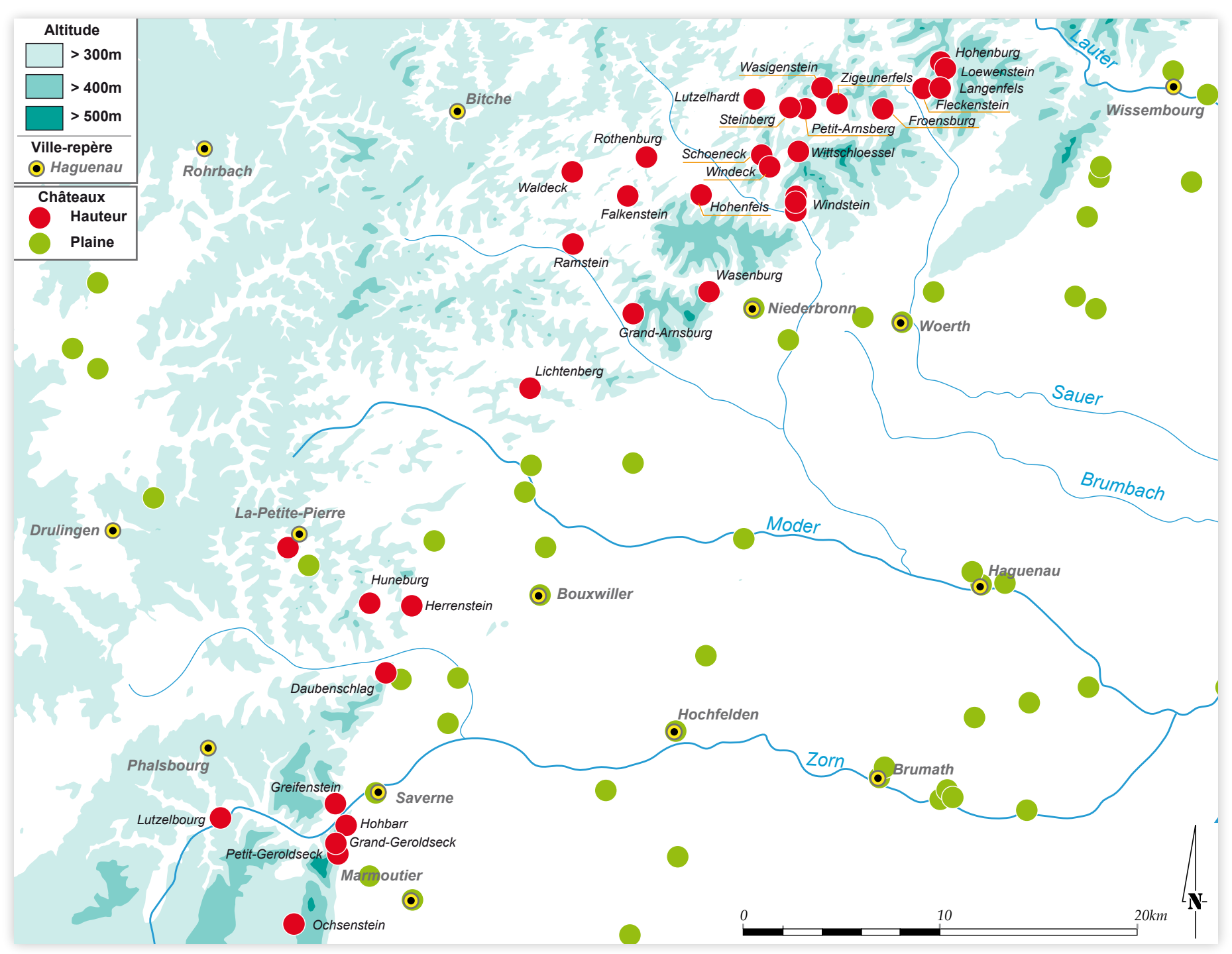

Figure 3 : carte de localisation des châteaux.

Nous avons également repris la liste des fortifications romaines de l'antiquité tardive, extraite du mémoire de maîtrise de Gertrud Kuhnle sur les sites des vallées du Rhin supérieur et du Haut-Rhin [4]. Il y a là une dizaine de mentions qui nous concernent mais que nous présenterons juste pour mémoire, ces vestiges devant être revisités pour en établir les contours au regard des connaissances actuelles (fig. 2).

La liste des châteaux est issue de la mise en commun de nombreux ouvrages qui y sont consacrés, validée par un travail collectif réunissant les castellologues alsaciens [5]. Nous considérerons ici tous les châteaux médiévaux conservés ou cités par des textes, quels que soient leur date de construction (entre 1000 et 1500), leur type (motte,

[4] KunnLE 1991. Ces données sont reprises et parfois complétées dans la CAG 67 (FLotTÉ \& FuCHS 2000).

[5] La carte générale issue de ce travail de validation (à l'échelle de toute la région) devrait faire l'objet d'une publication sur le site web de I'Atlas historique de I'Alsace, piloté par le CRESAT à l'université de Mulhouse. château de pierre...), leur possesseur (comte ou seigneur local...), etc. En revanche, nous ferons la différence entre les châteaux de plaine et ceux que nous avons appelés de hauteur, en ne retenant évidemment que ces derniers pour notre présente analyse, aux fins de comparaison avec les enceintes (fig. 3). Ces châteaux de hauteur, dont il n'existe pas de définition académique, sont ceux qui sont situés sur un promontoire (mont, plateau...) dominant largement les villages et villes alentours, soit dans le contexte particulier des Vosges du Nord, au-dessus de $250 \mathrm{~m}$ d'altitude. Dans cette sous-région, d'ailleurs, ces châteaux sont le plus souvent construits sur des barres rocheuses étroites et aux parois verticales, dont le Fleckenstein ou le Hohbarr sont les plus connus (fig. 4). Dans certains cas, ces sites comprennent physiquement deux, parfois trois châteaux sur des promontoires séparés mais très proches, comme le Windstein (Alt, Neu et Mittel), correspondant à des entités familiales et chronologiques comptabilisées par les historiens comme autant de châteaux. Dans notre cas, toutefois, qui vise à cerner des emprises de sites, nous avons réuni 
sous un seul vocable ces lieux doubles ou triples. Au total, notre liste comprend 28 sites [6].

Superposés sur une même carte, ces trois corpus, réunissant 60 entités, livrent deux ensembles bien séparés, avec un groupe de sites dans la partie nord, entre Niederbronn et Wissembourg, et un second entre Bouxwiller et Saverne, au sud. Le premier, avec environ 2/3 des occurrences, est le plus étoffé. Entre les deux se détache un espace quasi vide de tout vestige défensif ancien, sauf le Scheibenberg et le Hochkopf pour les enceintes et le Lichtenberg pour les châteaux (fig. 5). Ce sont ces deux groupes que nous analyserons ici de façon privilégiée.

\section{DONNÉES TOPOGRAPHIQUES}

Les Vosges sont un massif ancien aux formes émoussées, comme son homologue de la Forêt Noire en rive droite du Rhin. Formée dans son état actuel par le biais de la poussée des Alpes, les sommets les plus élevés se situent dans sa partie sud, autour de $1400 \mathrm{~m}$. Cette altitude diminue progressivement vers le nord, avec des hauteurs moyennes

[6] À cet ensemble, il faudrait ajouter une petite dizaine de sites immédiatement au-delà de la frontière avec le Palatinat, ces châteaux appartenant historiquement aux mêmes entités (par exemple, I'abbaye de Wissembourg pour le nord de la Lauter ou le comte palatin pour l'Alsace). Pour des questions de comparaison de corpus, nous n'en tenons toutefois pas compte, les recherches sur les enceintes plus anciennes dans cette partie du Palatinat n'ayant pas été retenues par Maxime Walter.
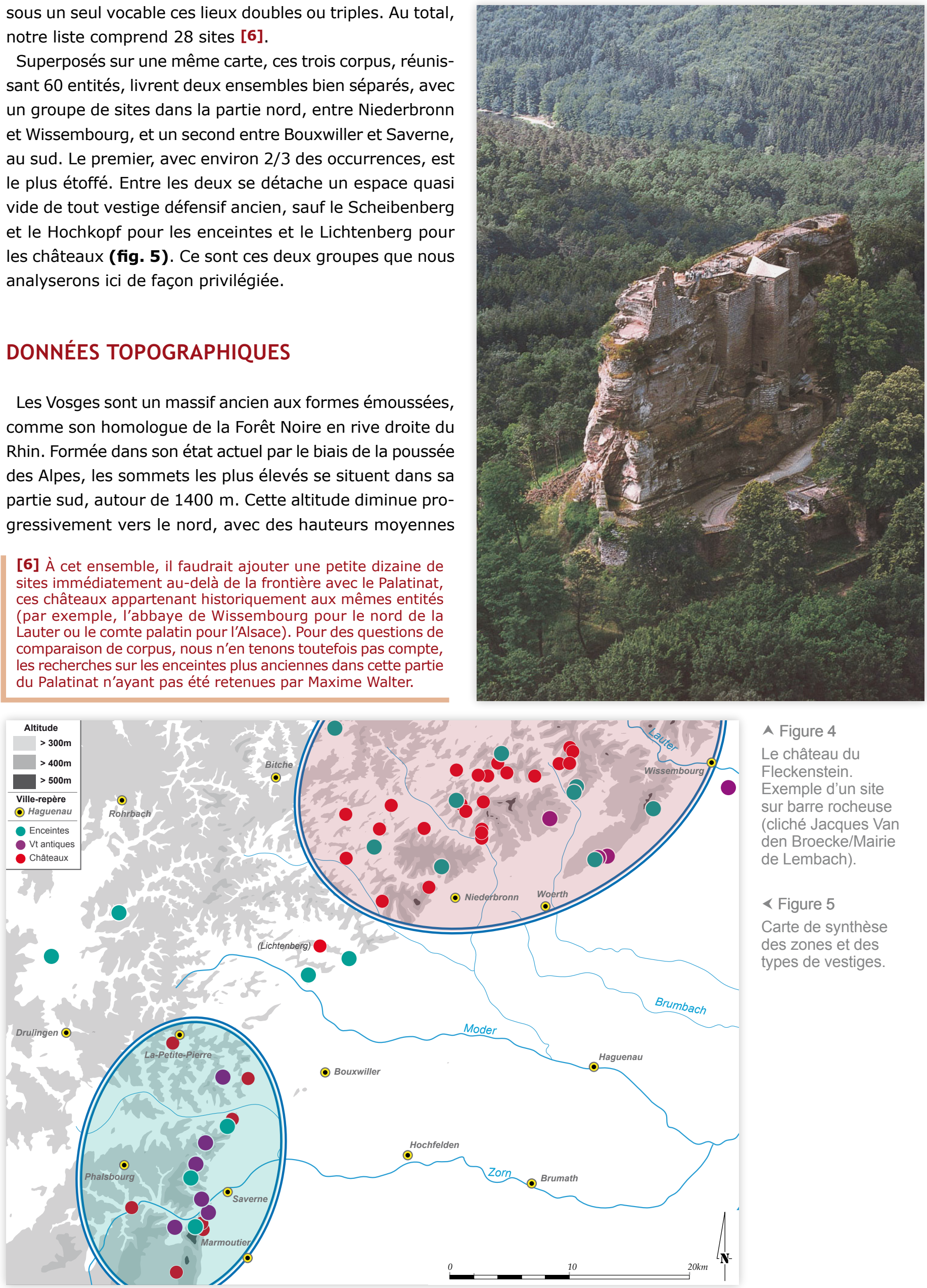

A Figure 4

Le château du

Fleckenstein.

Exemple d'un site sur barre rocheuse (cliché Jacques Van den Broecke/Mairie de Lembach)

$<$ Figure 5

Carte de synthèse des zones et des types de vestiges. 


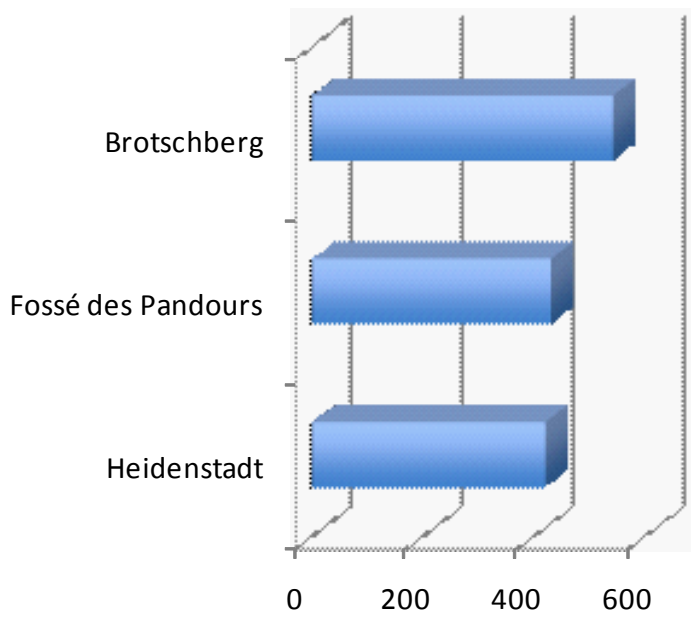

Figure 6

Tableau et graphique des altimétries des sites de la zone sud.

\begin{tabular}{|c|c|c|c|c|}
\hline & & COMMUNE & SITE & ALTITUDE \\
\hline & 1 & Ernolsheim/Saverne & Heidenstadt & 420 \\
\hline 岂 & 2 & Saverne & Fossé des Pandours & 430 \\
\hline$\overline{\mathrm{U}} \overline{\mathrm{O}}$ & 3 & Haegen & Brotschberg & 542 \\
\hline & & & Moyenne & 464 \\
\hline & 1 & Lutzelburg/Zorn & Lutzelburg/Zorn & 313 \\
\hline 5 & 2 & La-Petite-Pierre & La-Petite-Pierre & 350 \\
\hline$\sim$ & 3 & Saverne & Greiffenstein & 360 \\
\hline$\frac{w}{9}$ & 4 & Neuwiller & Herrenstein & 401 \\
\hline 오 & 5 & Ernolsheim & Daubenschlag & 405 \\
\hline (7) & 6 & Dossenheim/Zinsel & Huneburg & 415 \\
\hline 爻 & 7 & Saverne & Haut-Barr & 440 \\
\hline 屯্ড & 8 & Haegen & Geroldseck (Pt) & 464 \\
\hline 这 & 9 & Haegen & Geroldseck (Grd) & 473 \\
\hline$\perp$ & 10 & Reinhardtsmunster & Ochsenstein & 584 \\
\hline & & & Moyenne & 421 \\
\hline & & & Moyenne générale & 431 \\
\hline
\end{tabular}

de 300 à $500 \mathrm{~m}$ dans notre fenêtre d'étude, y formant des sortes de collines dominant la plaine entre 200 à $150 \mathrm{~m}$ du sud (Saverne) au nord (Wissembourg). La rupture topographique est toutefois plus marquée côté alsacien que Iorrain, avec une altitude de 250-300 m autour de Bitche. Cet ensemble est aujourd'hui peu urbanisé et couvert d'une forêt assez dense.

Nos deux groupes de systèmes défensifs devraient donc se situer logiquement dans des altitudes moyennes différentes. C'est effectivement le cas, mais dans des proportions moindres qu'attendues avec $430 \mathrm{~m}$ en moyenne pour le groupe sud et $380 \mathrm{~m}$ pour celui du nord. Dans le détail, nous observons aussi des nuances significatives entre les groupes (fig. 6-7). Les enceintes du sud sont plus élevées que celles du nord (464-385 m) alors que les châteaux y sont dans la moyenne générale, (433-382 m). Par ailleurs, le groupe sud occupe les sommets les plus élevés dans l'absolu, parfois au-delà de $500 \mathrm{~m}$, alors qu'au nord, la cote des $400 \mathrm{~m}$ est

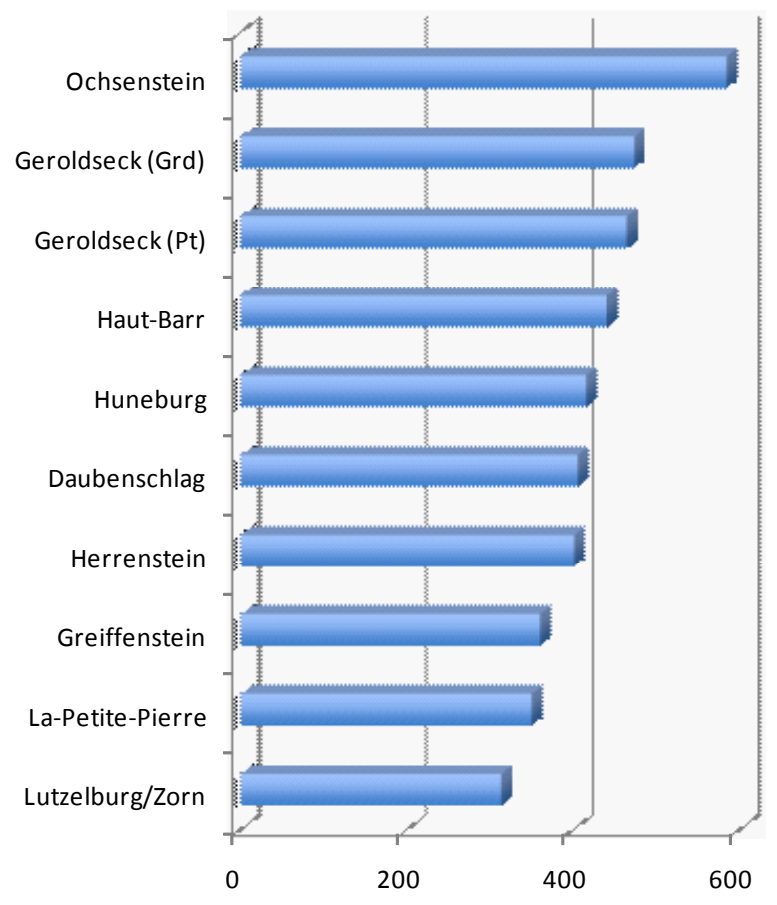

rarement atteinte. En somme, la recherche des sommets les plus élevés, qu'il s'agisse de châteaux ou d'enceintes, ne paraît pas être une démarche systématique.

La répartition spatiale des deux groupes livre d'autres pistes de réflexion. Au nord, I'ensemble des sites est réparti sur un large espace entre la plaine d'Alsace et le plateau lorrain (fig. 8). Les enceintes sont en général très éloignées les unes des autres, mais en même temps disposées le long d'arêtes SW-NE. Les châteaux, ici bien plus denses, sont mieux regroupés, dont un ensemble entre le Loewenstein et le Lutzelhardt paraît même organisé sur l'axe WE de la vallée du Steinbach. Par ailleurs, même dans ce groupe, de grandes plages de hauteurs sont inoccupées. En termes de topographie brute, le choix des sites paraît très divers d'un lieu à l'autre. Si quelques châteaux sont positionnés sur de belles éminences dominant une vallée (Windeck, Schoeneck ou les Windstein...), d'autres sont au contraire comme terrés au fond d'une reculée (Wasigenstein, Froensbourg, Wittschloessel...).

Le groupe sud est quant à lui organisé sur l'axe NS de I'arête vosgienne côté plaine d'Alsace (fig. 9). Les trois enceintes reconnues se répartissent régulièrement, alors que les châteaux sont là également partiellement groupés sur une arête au sud de Saverne. Si trois d'entre eux sont positionnés à l'entrée de vallées (Haut-Barr, Greifenstein pour la Zorn, Daubenschlag pour la Zinsel du sud), leur emplacement réel privilégie toutefois le versant plaine.

La position des sites en regard des cols et voies de passage se différencie également (fig. 10). Pour le groupe nord, d'une façon générale, les promontoires s'avançant vers les vallons, a priori propices à la surveillance de passages naturels, ne sont pas privilégiés. Tous les sites de 


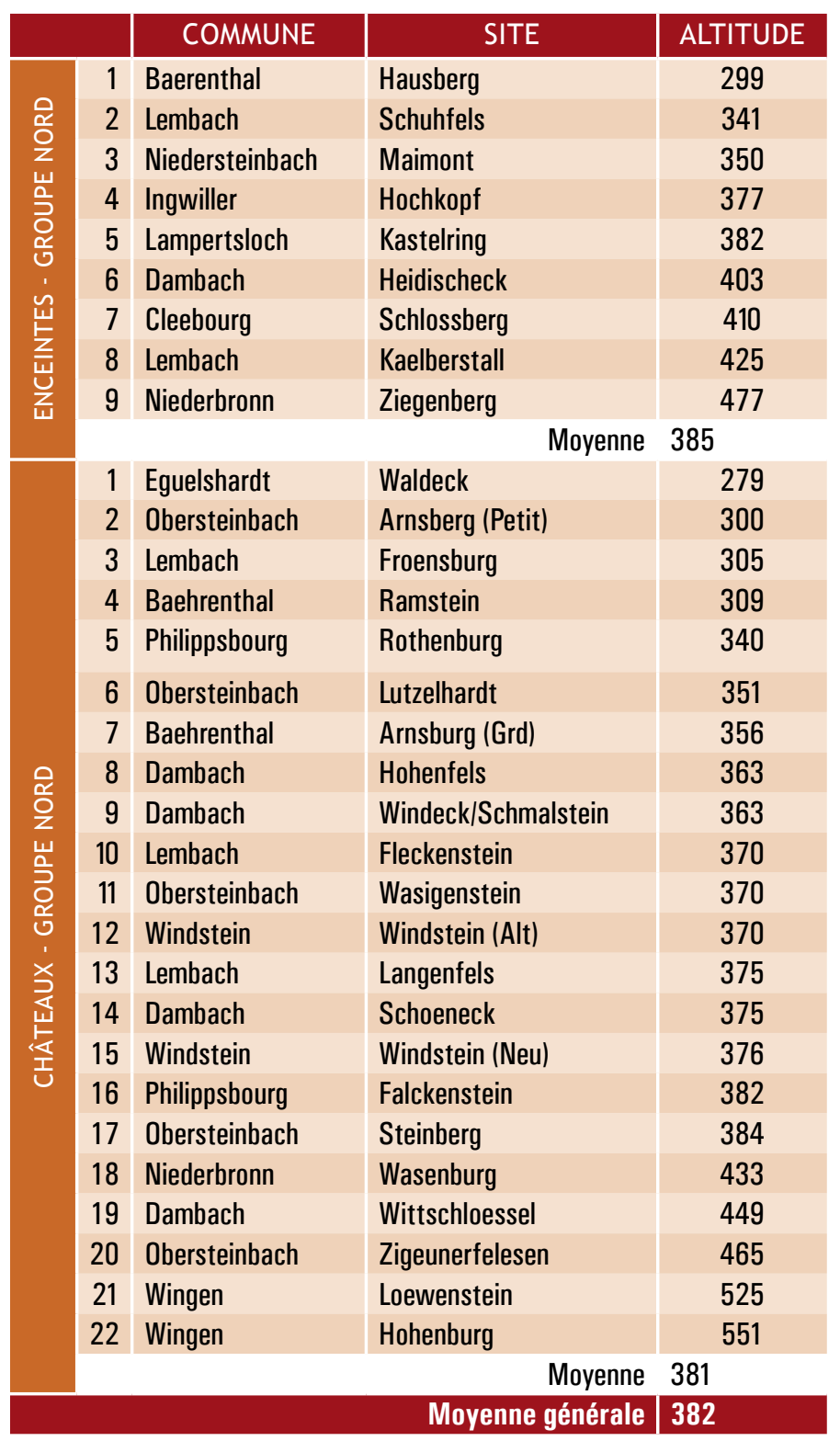

Figure 7

Tableau et graphique des altimétries des sites de la zone nord.

châteaux sont en retrait par rapport aux deux vallées principales. Les enceintes en revanche sont mieux placées par rapport à des voies SW-NE, comme Lembach-Wingen et Ingwiller-Wissembourg, mais à l'instar des châteaux, leur position par rapport aux cols actuellement aménagés n'est pas significative. Pour le groupe sud, en revanche, la densité des sites fortifiés paraît centrée sur le passage le plus important qu'est le col de Saverne, mais dont seul le site du Fossé des Pandours a un contact direct.

\section{DONNÉES DE PEUPLEMENT}

Le paramétrage des enceintes du groupe nord avec les données d'une occupation générale est malaisé, en raison de nos maigres connaissances sur l'occupation des espaces
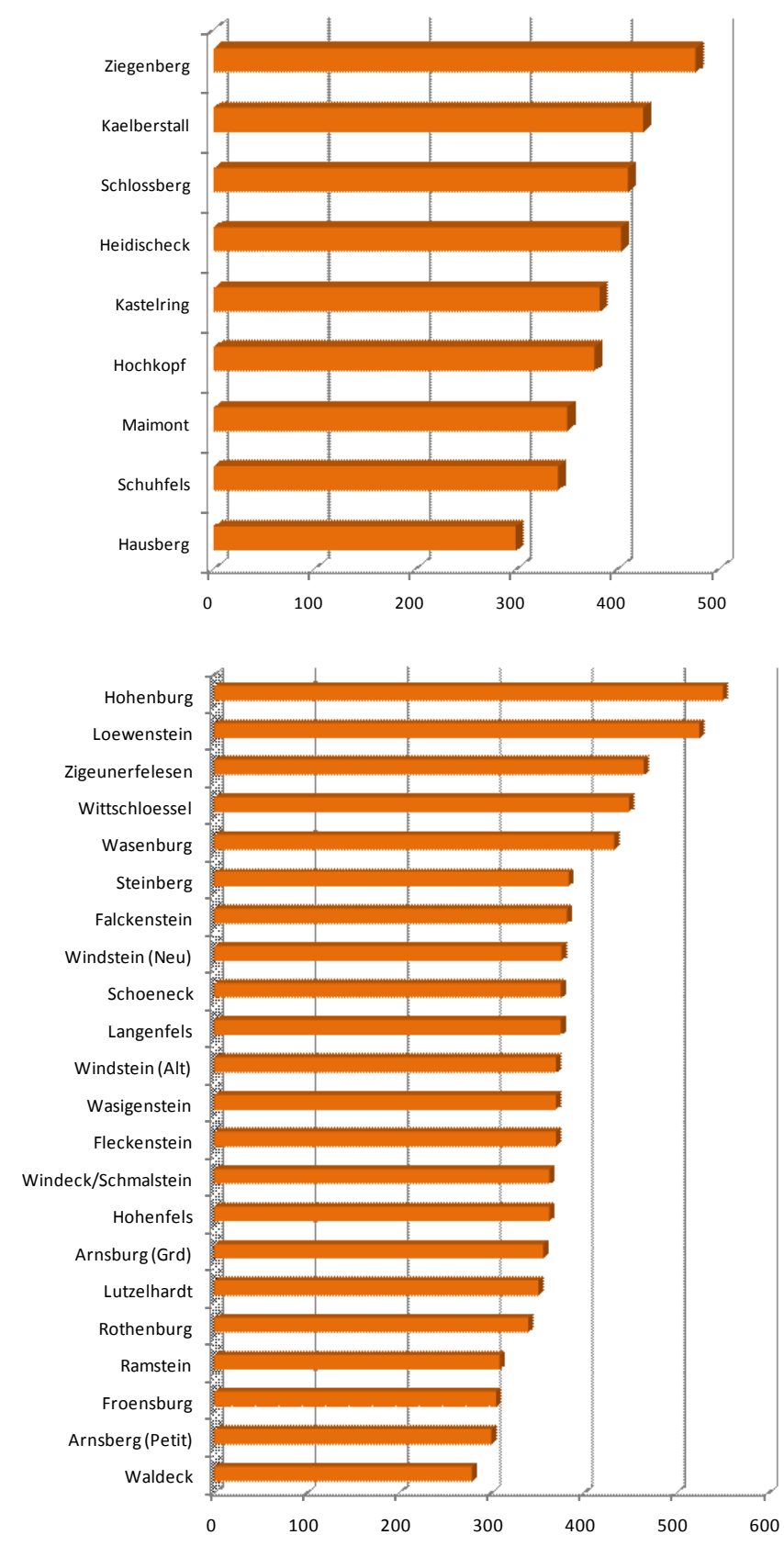

concernés [7]. Mais on y connait quelques nécropoles tumulaires et, pour l'Antiquité au moins, des sanctuaires (Lembach, Langensoultzbach (?), Wasenbourg) et des dépôts monétaires (Lembach), non loin des petites agglomérations du Piémont à Niederbronn ou Wissembourg-Altenstadt. Pour le groupe sud, les vestiges d'une occupation protohistorique et romaine sont plus fermement établis aux abords du col de Saverne avec des habitats de hauteur (Wasserwald, Uspann) et des cimetières (stèles-maisons de l'Eselfurt), en liaison là aussi avec une agglomération de plaine (Tres Tabernae).

[7] Les données générales sont reprises de Flotté-Fuchs, 2000. On consultera ausi les cartes de l'Atlas historique de I'Alsace (Protohistoire et Antiquité) qui complètent et précisent certains points. 


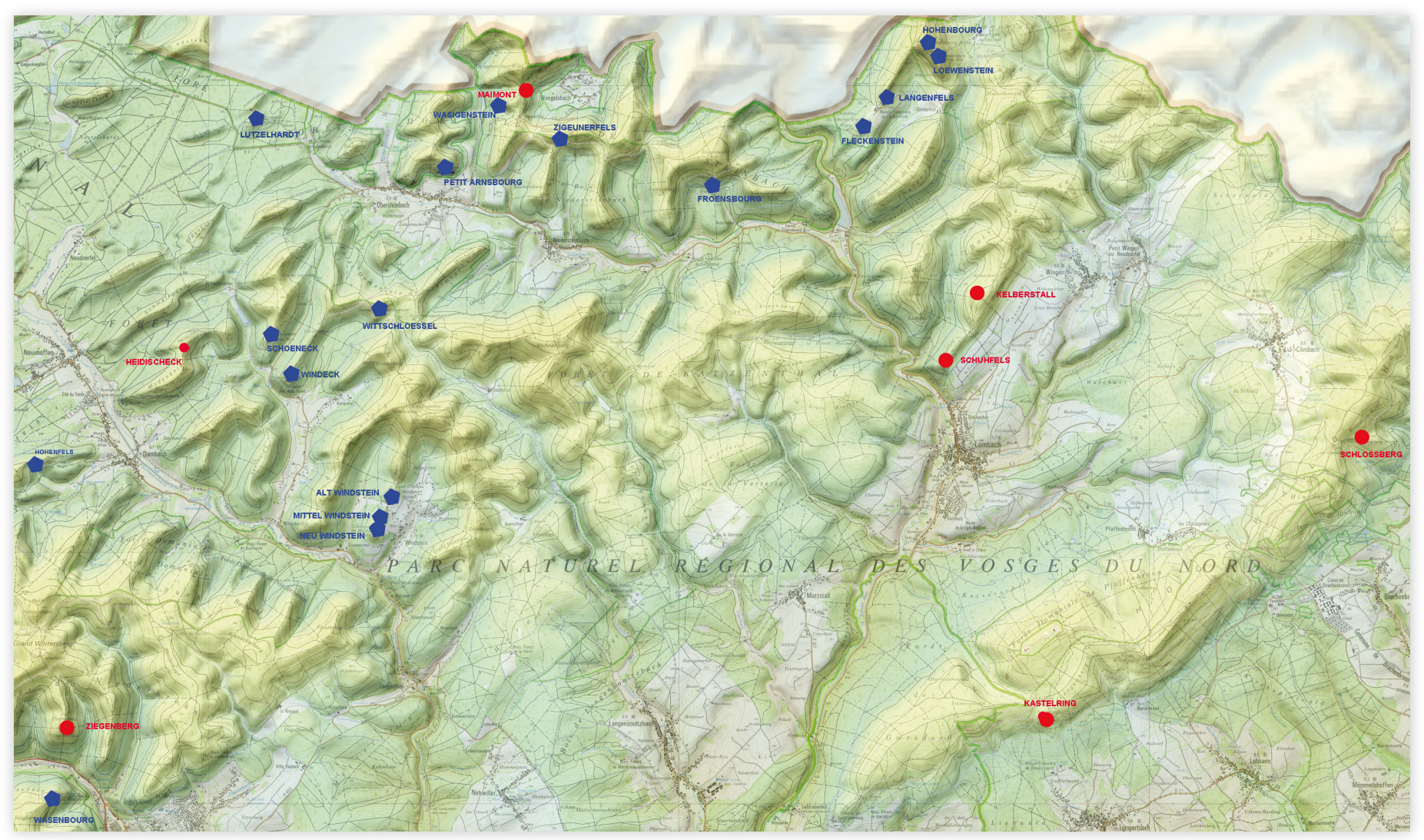

Figure 8

Carte topographique de la zone nord, avec enceintes et châteaux.

Pour le Moyen Âge, le contexte général est mieux connu [8]. Le mouvement de création de châteaux est animé par l'empereur, l'évêque de Strasbourg (DaubenschlagWarthenberg) et quelques familles comtales (Hunebourg) pour les $\mathrm{X}^{\mathrm{e}}$-XII ${ }^{\mathrm{e}}$ siècles, relayée par la petite noblesse au XIII ${ }^{\mathrm{e}}$ siècle. Il s'intègre le plus souvent dans un vaste processus de peuplement, parfois adossé à des abbayes dont les châtelains jouent le rôle d'avoués (protecteurs laïcs) mais également à des villes et bourgs aux fonctions politiques et économiques (marchés) complémentaires. Pour notre secteur, à la différence des Vosges du Sud où villes et abbayes innervent le tissu des vallées vosgiennes (Masevaux, Thann/ Saint-Amarin, Guebwiller/Murbach, Munster), I'essentiel des sites conventuels et urbains se sont développés le long de la route du Piémont. Seuls quelques bourgs de petite taille (Lichtenberg, La-Petite-Pierre) ou prieurés (Eschbourg, Sturtzelbronn) ont été édifiés dans l'espace des moyennes montagnes (fig. 11). Il faudrait également confronter la géographie des châteaux avec l'histoire du peuplement rural, mais nous manquons ici d'une synthèse fiable.

En partant de ces divers indices, on peut conclure sur les relations entre châteaux et peuplement. Pour le groupe sud, comme dans le cas des enceintes, nous nous situons dans une zone à forte valeur ajoutée, avec des châteaux isolés sur les hauteurs mais dominant un piémont densément occupé, entre Marmoutier et Neuwiller. Pour le groupe nord, en revanche, nos sites sont établis dans un espace de peuplement très lâche. Plusieurs châteaux (Loewenstein, Froensbourg, Wasigenstein, Wittschloessel, etc.) sont même complètement isolés, à une bonne heure de marche du premier village. Certains, comme le Wasigenstein, n'ont jamais développé de seigneurie, une forme d'organisation politique et économique indispensable à la fidélisation d'une population. La densité des châteaux y est même inversement proportionnelle à celle du peuplement : alors que la règle générale est d'avoir un château pour un ou deux villages, le ratio est ici de 2, 3 (Lembach, Windstein) voire 4 à 5 (Dambach, Obersteinbach) châteaux par commune actuelle, soit une configuration exceptionnelle (fig. 12) [9].

[8] Biller-Metz, 1992 ; Pottecher et al. 2015, carte des châteaux (p. 179) et des villes/centres de pouvoir (villes, palais, abbayes) pp. 214-215, pour la période 1000/1250. Pour le détail de l'histoire des sites, on lira avec intérêt un article qui aborde les deux versants de la frontière de I'Alsace du nord (Kill-Rudrauf, 2010).

[9] Nous avons élaboré notre carte fig. 12 à partir de l'organisation communale actuelle, ce qui bien entendu n'est pas sans danger. Mais si au moment de la plus forte dynamique des constructions castrales, soit le $\mathrm{XIII}^{\mathrm{e}} \mathrm{S}$., les terroirs ne sont pas encore entièrement délimités comme aujourd'hui, les communautés d'habitants, avec église paroissiale et institutions spécifiques, sont pour l'essentiel constituées dans des proportions proches de notre géographie communale. Même si l'outil est imparfait, une estimation du ratio nombre de communautés d'habitants/proportions des équipements spécifiques - ici, le château - vaut comme indice d'une histoire du peuplement. 

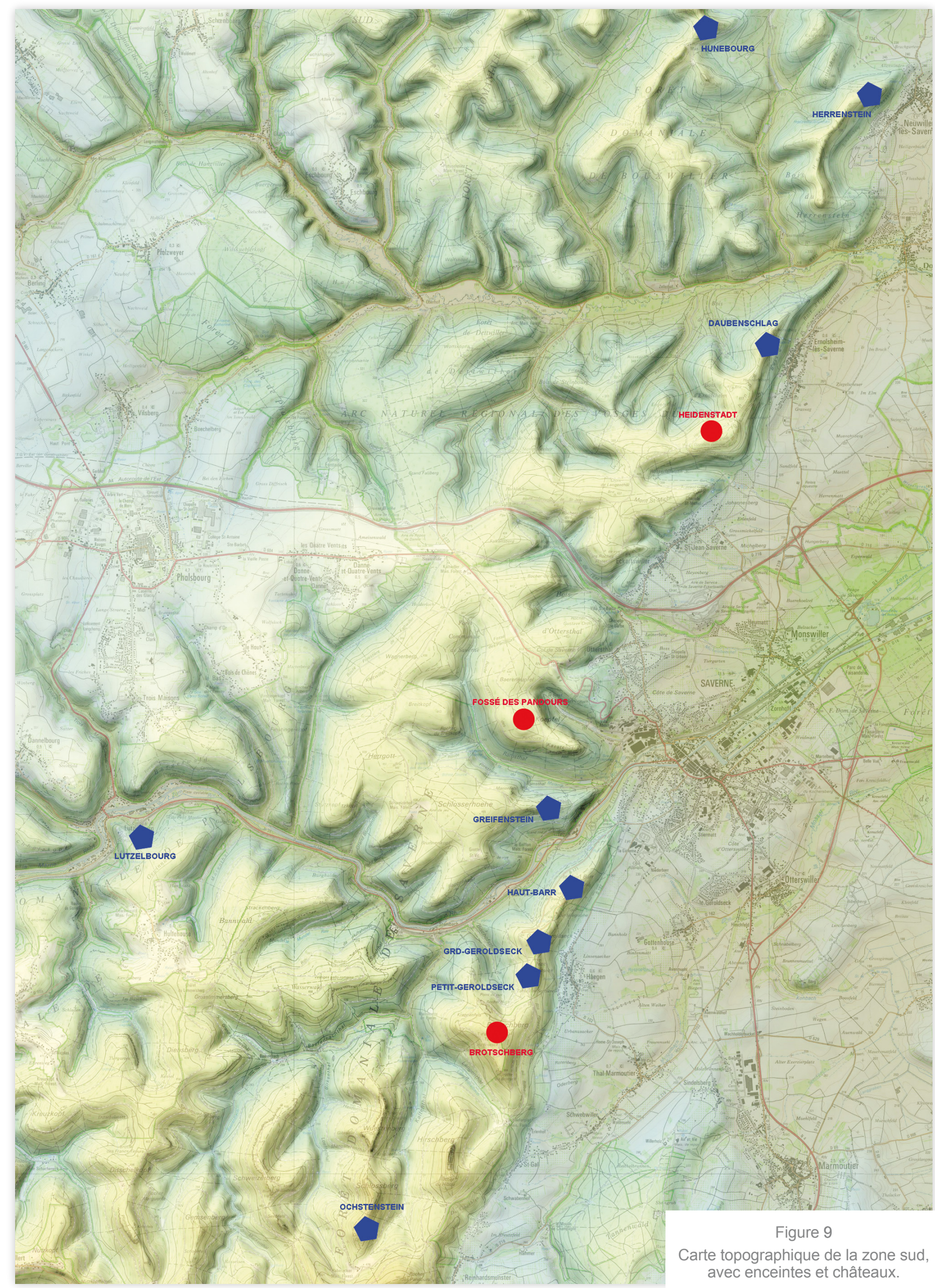

Figure 9

Carte topographique de la zone sud, avec enceintes et châteaux. 
Même si nos connaissances du peuplement médiéval pour cette zone restent imprécises, on peut poser comme hypothèse forte la présence d'un dense réseau de châteaux dans un espace de peuplement lâche. Hors les réserves forestières et certaines activités industrieuses comme la verrerie ou la métallurgie (mais mal cernées pour notre période) [10], l'attraction économique de la zone est des plus réduite. La question est évidemment de savoir pourquoi ces choix d'implantation. La situation actuelle inviterait à y reconnaître une problématique de zone-frontière, mais ceci n'a pas de sens pour le Moyen Âge. De même, l'idée d'une continuité des occupations sur un même lieu peut être écartée, les châteaux étant sauf exception (Wasenbourg, et peut être Griffon et Hohbarr) construits sur des sites vierges. En revanche, tous ces châteaux (sauf le Fleckenstein), appartiennent au XIII ${ }^{\mathrm{e}}$ siècle et sont en partie au moins construits par des ministériaux. Il s'agit là d'une catégorie d'administrateurs impériaux ou épiscopaux d'origine servile qui, en particulier lors de la vacance du pouvoir impérial dans la seconde moitié du XIII ${ }^{\mathrm{e}}$ s., ont conquis leur autonomie, formant dès lors les cadres de la petite noblesse. Le château servant ici comme ailleurs d'ascenseur social, le choix d'une zone à peuplement lâche, sans contestation d'un seigneur déjà bien établi, pourrait ainsi être un moteur de ces implantations. Cette hypothèse est confortée par la relative courte durée de vie de ces sites : sur les 22 châteaux de notre groupe nord, six seulement semblent encore actifs après 1500 , deux étant fortement réadaptés aux armes à feu (Schoeneck, Hohenbourg) et quatre autres ayant fait l'objet de réaménagements minimes à la Renaissance (Fleckenstein, Froensbourg, Windstein, Petit Arnsberg) [11].

[10] L'industrie du verre tout comme celle du fer (et sa transformation en fonte) ne sont attestées à grande échelle dans les Vosges du Nord qu'à partir de la fin de l'époque moderne avec des productions renommées (verrerie Lalique, usines de Dietrich). Pour l'instant, les indices reconnus d'activités industrieuses dans ces domaines-là sont trop ténus pour permettre d'envisager une attractivité proto-industrielle de cette région.

[11] BRONNER \& SCHWIEN, à paraître.

Figure 10 : carte de la voirie et des cols actuels.

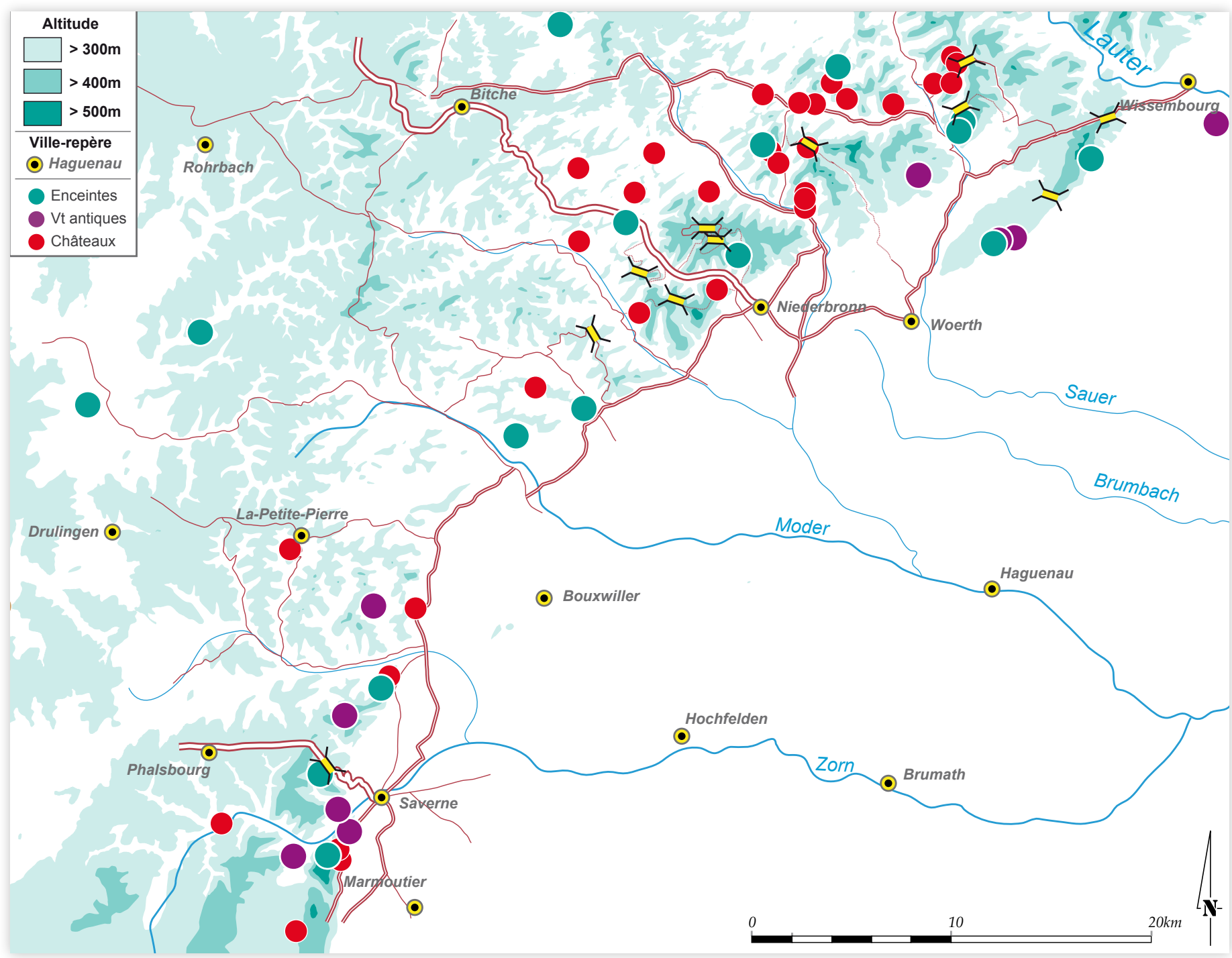




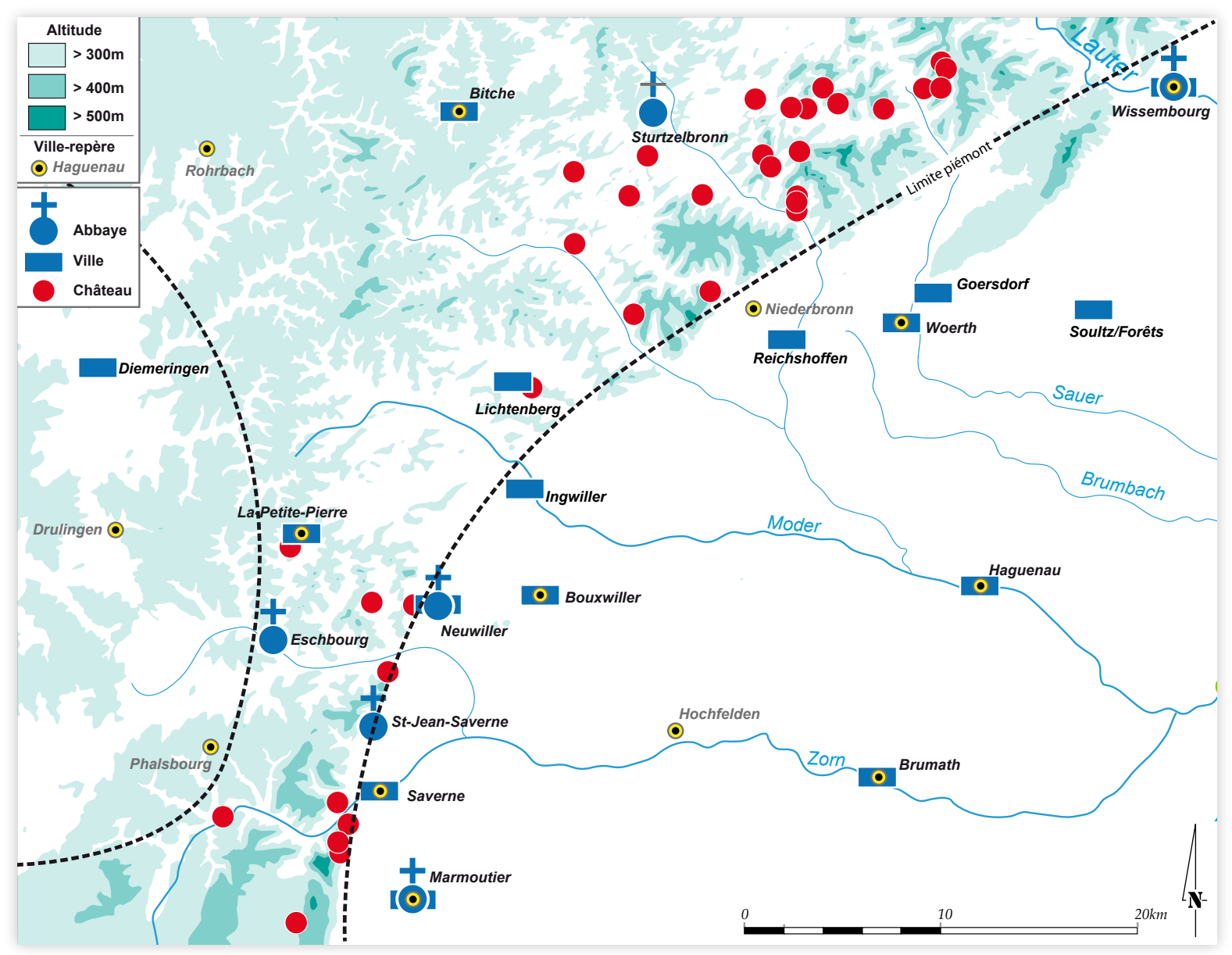

A Figure 11 : carte des abbayes et villes du piémont.

$\checkmark$ Figure 12 : carte de la densité des sites de châteaux sur les territoires des communes actuelles.

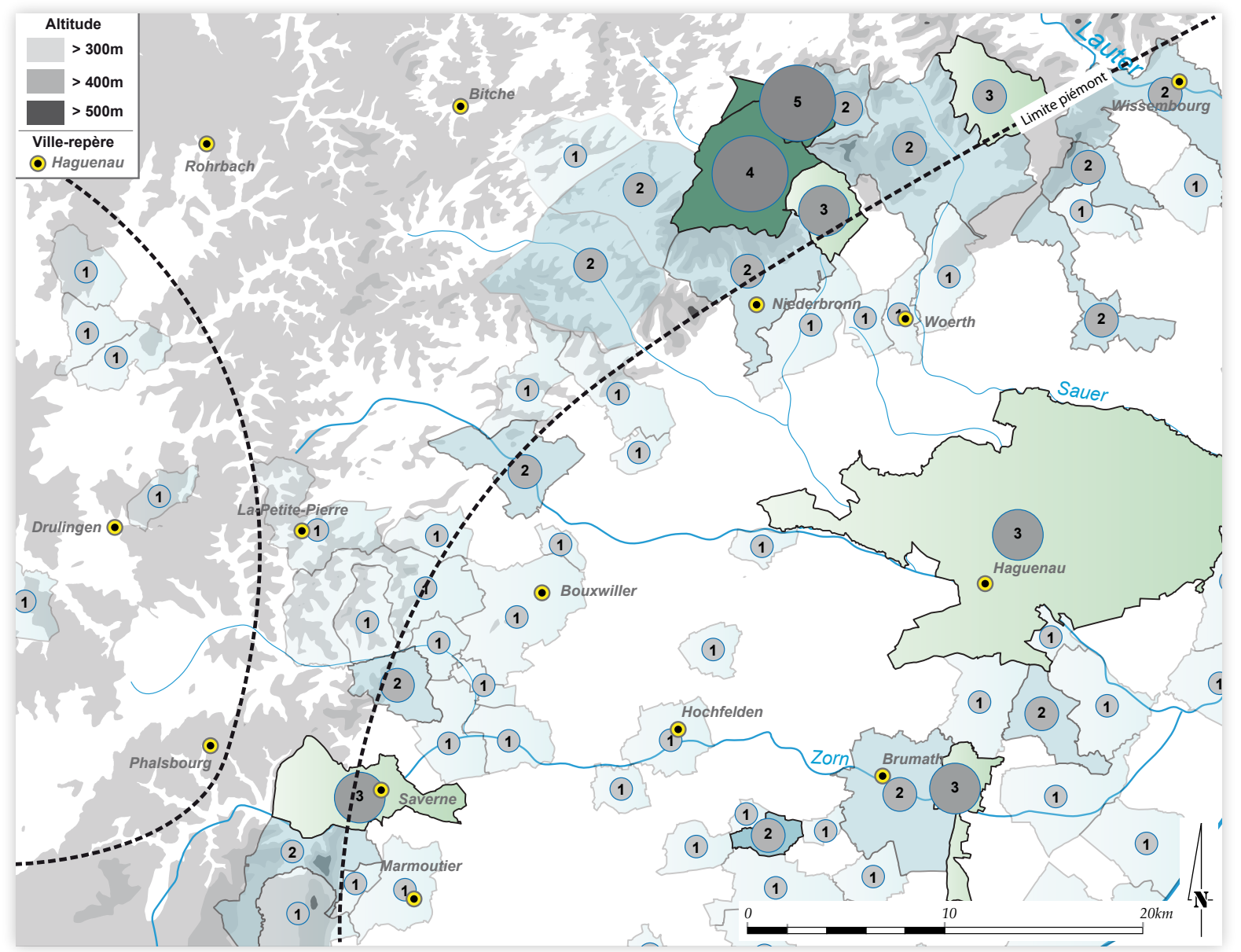




\section{CONCLUSION}

À la question initiale des raisons du choix de l'implantation d'un site défensif de hauteur en un lieu donné, les réponses sont multiples. Nos argumentaires sont fragiles du fait d'un corpus toujours incomplet, tant en termes de sites de hauteur que des zones d'activité ou d'habitat, même si nous cernons un peu mieux les données de la fin du Moyen Âge. Par ailleurs, les arguments « de bon sens » sur le perchement idéal au sommet d'un promontoire, la position par rapport à un col ou un point de passage, la permanence des lieux, etc., doivent tous être sinon remisés, du moins confrontés à une mise en situation générale. Ainsi, si dans nos deux zones, certains lieux « idéaux » ont été occupés, il existe de nombreux autres promontoires qui auraient pu faire l'affaire et qui sont inoccupés (de façon incertaine pour les enceintes et de façon sûre pour les châteaux). De même, nous avons observé des situations opposées entre un groupe nord médiéval, dont c'est le caractère « désertique » qui a pu attirer les constructeurs de châteaux (avec même des implantations dans des reculées plutôt qu'en tête de vallon) et un groupe sud où c'est la densité générale de la population (et donc des centres de pouvoir) qui a pu être le moteur. Enfin, en comparant les lieux d'implantation des enceintes « collectives » et des châteaux, on observe des géographies très différentes, témoignant de stratégies topo-économico-politiques spécifiques, même si le sens nous en échappe.

La fenêtre choisie n'est sans doute pas idéale et d'autres conclusions auraient pu être tirées de la confrontation des sites fortifiés des vallées vosgiennes du Sud. Mais nous espérons que notre rapide survol aura au moins invité à dépasser des problématiques fondées sur une collection de sites pour aborder des territoires sur la longue durée.

\section{BIBLIOGRAPHIE}

Biller, Thomas \& MeTZ, Bernhard, 1991, « Anfänge der Adelsburg im Elsass in ottonischer, salischer und frühstaufischer Zeit », dans Horst Wolfgang Böhme (éd.), Burgen der Salierzeit, Sigmaringen, p. 245-284 (avec une liste des premières mentions jusqu'en 1200).

Böнme, Horst Wolfgang (éd.), 1991, Burgen der Salierzeit. Teil 1 : in den nördlichen Landschaften des Reiches; Teil 2 : In den südlichen Landschaften des Reiches, Sigmaringen.

Bronner, Guy \& Schwien, Jean-Jacques, à paraître, « Le devenir du château après 1500 en Alsace », dans Nicolas Faucherre, Delphine Gautier \& Hervé Mouillebouche (éd.), Fortifier sa demeure du XVI au XVIII siècle, (Colloque de Bellecroix 5), Chagny.

Flotté, Pascal \& Fuchs, Matthieu (éd.), 2000, Carte archéologique de la Gaule. Bas Rhin, Paris.

Himly, François-Jacques, 1970, Atlas des villes médiévales d'Alsace, Strasbourg.

Kill, René \& Rudrauf, Jean-Michel, 2010, « Découverte de sites fortifiés et de rochers aménagés médiévaux dans les Vosges du Nord depuis le début du XXe siècle », Châteaux-forts d'Alsace 11, p. 61-80.

KILL, René, 2012, L'approvisionnement en eau des châteaux forts de montagne alsaciens, Saverne.

KunnLe, Gertrud, 1991, Les fortifications romaines de l'antiquité tardive des vallées du Rhin supérieur et du Haut-Rhin, Mémoire de maîtrise (faculté des Sciences historiques), Université des Sciences Humaines de Strasbourg, 2 vol.

Pottecher, Marie, Schwien, Jean-Jacques, Meyer, Jean-Philippe \& Freund-Lehmann, Alexandre (éd.), 2015, L'Alsace au cœur du Moyen Âge. De Strasbourg au Rhin supérieur (XI ${ }^{e}-X I I^{e}$ s.), Lyon.

Steuer, Heiko \& Bierbrauer, Volker (éd.), 2008, Höhensiedlungen zwischen Antike und Mittelalter von den Ardennen bis zur Adria, Berlin.

Walter, Maxime, 2016, «Les sites de hauteur du massif Vosgien. Actualisation des données et modalités d'implantation », Archimède 3, p. 20 à 36. 